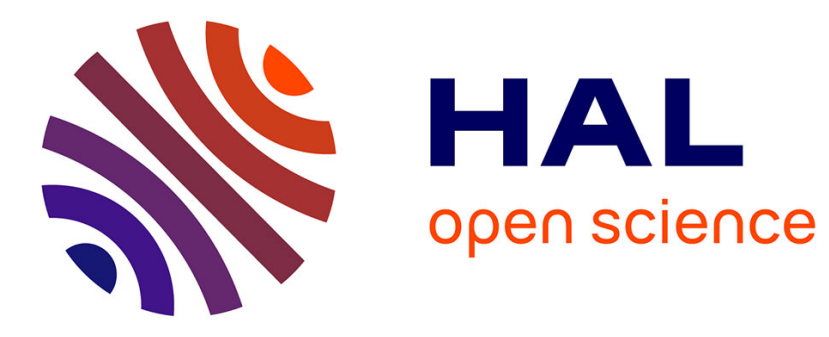

\title{
Prospective assessment of the endometrium in postmenopausal breast cancer patients treated with fulvestrant
}

Leilani Morales, Patrick Neven, Dirk Timmerman, Hans Wildiers, Maja L. Konstantinovic, Marie-Rose Christiaens, Peter N. Tan, Robert Paridaens

\section{To cite this version:}

Leilani Morales, Patrick Neven, Dirk Timmerman, Hans Wildiers, Maja L. Konstantinovic, et al.. Prospective assessment of the endometrium in postmenopausal breast cancer patients treated with fulvestrant. Breast Cancer Research and Treatment, 2008, 117 (1), pp.77-81. 10.1007/s10549-0080248-2 . hal-00535317

\section{HAL Id: hal-00535317 https://hal.science/hal-00535317}

Submitted on 11 Nov 2010

HAL is a multi-disciplinary open access archive for the deposit and dissemination of scientific research documents, whether they are published or not. The documents may come from teaching and research institutions in France or abroad, or from public or private research centers.
L'archive ouverte pluridisciplinaire HAL, est destinée au dépôt et à la diffusion de documents scientifiques de niveau recherche, publiés ou non, émanant des établissements d'enseignement et de recherche français ou étrangers, des laboratoires publics ou privés. 


\title{
Prospective assessment of the endometrium in postmenopausal breast cancer patients treated with fulvestrant
}

\author{
Leilani Morales $\cdot$ Patrick Neven $\cdot$ Dirk Timmerman $\cdot$ \\ Hans Wildiers · Maja L. Konstantinovic · Marie-Rose Christiaens • \\ Peter N. Tan · Robert Paridaens
}

Received: 4 November 2008/Accepted: 4 November 2008/Published online: 2 December 2008

(C) Springer Science+Business Media, LLC. 2008

\begin{abstract}
This prospective study assessed the endometrial effects of fulvestrant, a pure estrogen-receptor antagonist, in postmenopausal women with breast cancer. This singlecenter study enrolled postmenopausal patients who had an intact uterus at baseline with progressive metastatic breast cancer on tamoxifen followed by an oral aromatase inhibitor (AI). Fulvestrant (250 mg) was administered every $28 \pm 3$ days via IM injection. Transvaginal ultrasonography (TVUS) was performed at baseline and after 3 months of therapy. Primary and secondary endpoints were changes from baseline in double endometrial thickness (DET) and uterine volume (UV), respectively. No interventions were performed on any asymptomatic uterine abnormalities that were detected at baseline. In total, 32 women were enrolled. Five patients had no repeat TVUS because of early progression before 3 months, leaving 27 evaluable patients for final analysis. After 3 months therapy, mean DET had
\end{abstract}

L. Morales $\cdot$ H. Wildiers $\cdot$ R. Paridaens $(\square)$

Department of Medical Oncology, University Hospital

Gasthuisberg, Herestraat 49, 3000 Leuven, Belgium

e-mail: robert.paridaens@uz.kuleuven.ac.be

L. Morales - P. Neven - D. Timmerman - M. L. Konstantinovic Department of Obstetrics and Gynecology, University Hospital Gasthuisberg, Leuven, Belgium

P. Neven - M.-R. Christiaens $\cdot$ R. Paridaens Department of Multidisciplinary Breast Center, University Hospital Gasthuisberg, Leuven, Belgium

M.-R. Christiaens

Department of Surgery, University Hospital Gasthuisberg, Leuven, Belgium

P. N. Tan

Erasmus Mundus Master Programme, Faculty of Medicine, Katholieke Universiteit Leuven, Leuven, Belgium significantly decreased by $23.08 \%(P=0.010)$. Mean UV also decreased by $10.88 \%$, although this change was not significant $(P=0.119)$. After 3 months of therapy, none reported vaginal bleeding, there were no changes noted in most of the uterine pathologies present at baseline and no new uterine abnormalities were detected. We observed that 3 months of fulvestrant treatment resulted in a significant decrease in endometrial growth and a non-significant decrease in UV in postmenopausal women with metastatic breast cancer previously exposed to tamoxifen and AIs. Furthermore, no new uterine pathologies were detected, indicating that fulvestrant behaves as a pure antiestrogen at the uterine level.

Keywords Breast cancer - Endometrium - Fulvestrant . Hormonal therapy · Ultrasonography · Uterus

\section{Introduction}

Since the time tamoxifen, a selective estrogen-receptor modulator has been shown to have an estrogen agonist effect on the uterus, the endometrial effects of anti-hormonal treatments for breast cancer have remained an issue of clinical concern. Tamoxifen administration is known to be associated with gynecological side effects such as endometrial hyperplasia, polyps and cancer [1-4]. Although most of these changes are benign, the wide range of abnormalities induced by tamoxifen has led to a number of gynecological interventions in symptomatic women to exclude malignant disease. The third generation aromatase inhibitors (AIs) which are increasingly used as an alternative or as an additional treatment to tamoxifen [5-7] do not have an estrogenic effect on the uterus and may even reverse tamoxifen-associated endometrial pathologies as 
shown in several studies [8-15]. The potential use of AIs specifically for the management of endometrial pathologies is promising [16].

Among the significant advances in endocrine therapy of breast cancer is the introduction of the selective estrogenreceptor antagonist, fulvestrant. In vitro and in vivo studies in animal models demonstrated that it does not have an agonist effect on estrogen-sensitive tissues and that it binds, blocks and increases degradation of the estrogen receptor [17]. Fulvestrant is now widely used in postmenopausal women with metastatic ER-positive breast cancer [18-21] in whom it has been shown to be as effective as the steroidal AI, exemestane, in second line anti-hormonal treatment [22]. Aside its demonstrated efficacy against metastatic breast cancer, little is known about its effect on other estrogen sensitive tissues. Studies that have assessed fulvestrant's effect on the uterus are limited [23]. In a phase 1 study of healthy postmenopausal volunteers whose endometrium was stimulated with ethinylestradiol, one single administration of fulvestrant at a dose of $250 \mathrm{mg}$ was shown to significantly inhibit the estrogen-stimulated thickening of the endometrium compared with placebo [24]. However, in a study of premenopausal patients with uterine fibroids awaiting hysterectomy, fulvestrant did not significantly alter fibroid volume or endometrial thickness or change endpoints such as endometrial histology or vaginal bleeding compared to goserelin [25]. Few case reports suggest the potential of fulvestrant as an effective therapy for patients with hormone receptor-positive uterine cancer [26, 27]. At least two clinical trials currently test fulvestrant against progestins for metastatic endometrial cancer. While fulvestrant is considered a pure antiestrogen, it is possible that it may have estrogen-like effects on other estrogen sensitive tissues such as the bone. This is another reason why fulvestrant's effect on other tissues is of interest.

To date, the effect of fulvestrant on the postmenopausal uterus and the endometrium in particular is not known. The aim of the present study was to determine any endometrial effects of fulvestrant in postmenopausal women with breast cancer, which is of paramount importance for any wider use of this drug, like in the adjuvant or even in the preventive setting.

\section{Patients and methods}

This prospective, single-center study enrolled non-hysterectomised postmenopausal patients with metastatic breast cancer, who previously received tamoxifen followed by an AI, and who were estimated to benefit from fulvestrant for at least 3 months. Fulvestrant $(250 \mathrm{mg})$ was administered every $28 \pm 3$ days via IM injection.
The study aimed at investigating the transvaginal ultrasonography (TVUS) changes occurring during the first 3 months of therapy, so that all patients had to undergo this investigation at baseline within the 2 weeks preceding the first fulvestrant administration, and to have a repeat examination after 3 months. The primary study-endpoint was the change in endometrial thickness (DET) and secondary endpoints were the changes in uterine volume (UV) and uterine abnormalities from baseline to after 3 months of therapy.

Two investigators (DT and MLK) performed TVUS at baseline and after 3 months of therapy. The uterus was completely assessed in sagittal and coronal planes with uterine size recorded in three diameters $\left(D_{1,2,3}\right)$. The volume (ml) was estimated as $\left(D_{1} \times D_{2} \times D_{3} \times 3.14\right) / 6$. DET was measured in the sagittal plane, from one endometrialmyometrial interface to another excluding intracavitary fluid. Endometrial abnormalities such as internal cysts and polyps were recorded. The presence of internal cysts was defined by visualization of more than one anechogenic area greater than $2 \mathrm{~mm}$. No interventions were performed on any asymptomatic uterine abnormalities that were detected at baseline.

The study was conducted according to the guidelines for clinical studies described in the Declaration of Helsinki [28]. The protocol was approved by the Ethical Committee for Clinical Studies of the University Hospitals Leuven, Belgium before commencement of the study and all participants provided written informed consent.

Statistical analysis was done with SPSS (Version 14). Paired $t$-tests were used to assess the statistical significance of any differences in DET and UV at baseline and after 3 months of therapy with $P<0.05$ considered significant.

\section{Results}

In total, 32 postmenopausal women with metastatic breast cancer were enrolled in this study. In five patients, treatment was discontinued early, prior to the 3-month re-evaluation due to disease progression. Twenty-seven completed all the planned investigations and are included in this final analysis. All had previously received tamoxifen followed by an AI. The mean age of the study population was 64 years and patients were on average 12 years past menopause. Table 1 shows the changes in DET and UV from baseline to 3 months of therapy. At baseline, the mean $( \pm \mathrm{SD})$ DET was $4.00 \pm 2.57 \mathrm{~mm}$ and UV was $41.89 \pm 32.70 \mathrm{~cm}^{3}$. After 3 months of therapy, mean DET had significantly decreased by $23.08 \%$ to $3.07 \pm 1.58 \mathrm{~mm}$ $(P=0.010)$. Mean UV also decreased by $10.88 \%$ to $37.33 \pm 21.96 \mathrm{~mm}^{3}$, although this change was not significant $(P=0.119)$. The change in DET and UV from 
Table 1 Changes in DET and UV from baseline to 3 months of therapy

\begin{tabular}{lrlll}
\hline & Mean & SD & SE & $P$ value \\
\hline DET (mm) & & & & \\
Baseline & 4.00 & 2.57 & 0.49 & \\
3 Months & 3.07 & 1.58 & 0.30 & \\
Difference & -0.92 & 1.74 & 0.33 & $P=0.010$ \\
UV $\left(\mathrm{cm}^{3}\right)$ & & & & \\
Baseline & 41.89 & 32.70 & 6.29 & \\
3 Months & 37.33 & 21.96 & 4.23 & \\
Difference & -4.56 & 14.69 & 2.83 & $P=0.119$ \\
\hline
\end{tabular}

Abbreviations: DET double endometrial thickness; $U V$ uterine volume

baseline to 3 months treatment for each individual patient is illustrated in Fig. 1.

In the few patients with uterine abnormalities at baseline, the changes observed at 3 months are summarized in Table 2 and are as follows: in five patients, there was no change in endometrial cysts; among two patients with polyps, polyp size decreased in one and slightly increased in the other; there were no changes in fibroids in two patients, while fibroid size decreased in one and increased in another; the size of hydrosalpinx increased in one case, and the size of metastatic ovarian deposits slightly decreased in another one. No new uterine abnormalities were detected after 3 months of therapy in any patient. It is also noteworthy that there were no episodes of vaginal bleeding reported during the entire study duration.

\section{Discussion}

To our knowledge, this is the first prospective study exploring the uterine effect of fulvestrant in postmenopausal breast cancer patients. The women in our study received prior endocrine therapy with tamoxifen followed by a steroidal or non-steroidal AI. In accordance with the well known atrophic effect of AIs on the endometrium, we observed at baseline a thin endometrium and a small uterine volume in most of the patients included in the present study. Indeed there were only seven patients with a DET thicker than $4 \mathrm{~mm}$, and only seven patients with a uterine volume greater than $40 \mathrm{~cm}^{3}$. Very remarkably, treatment with fulvestrant still led to a further decrease in endometrial thickness and uterine volume after 3 months as illustrated in Fig. 1. One of the issues inherent in a shortterm study like this is the probability that the uterine changes observed in our study may be due to a carry-over effect of prior AI therapy rather than a real fulvestrant effect. Given the short-term evaluation in our study and the absence of a wash-out period between prior AI therapy and succeeding fulvestrant therapy, the decrease in DET and UV on top of prior AI therapy cannot be attributed to fulvestrant alone with absolute certainty. However, it is clear that after 3 months of therapy with fulvestrant, there were no signs on TVUS of a stimulatory effect on the endometrium in contrast to what has been detected on TVUS after 3 months of tamoxifen therapy [8].

Aside from the decrease in DET and UV, we observed that most of the uterine pathologies detected at baseline did
Fig. 1 Changes in DET and UV from baseline to 3 months of therapy for each patient. Note: Each line represents each individual patient.

Abbreviations: DET, double endometrial thickness; UV, uterine volume
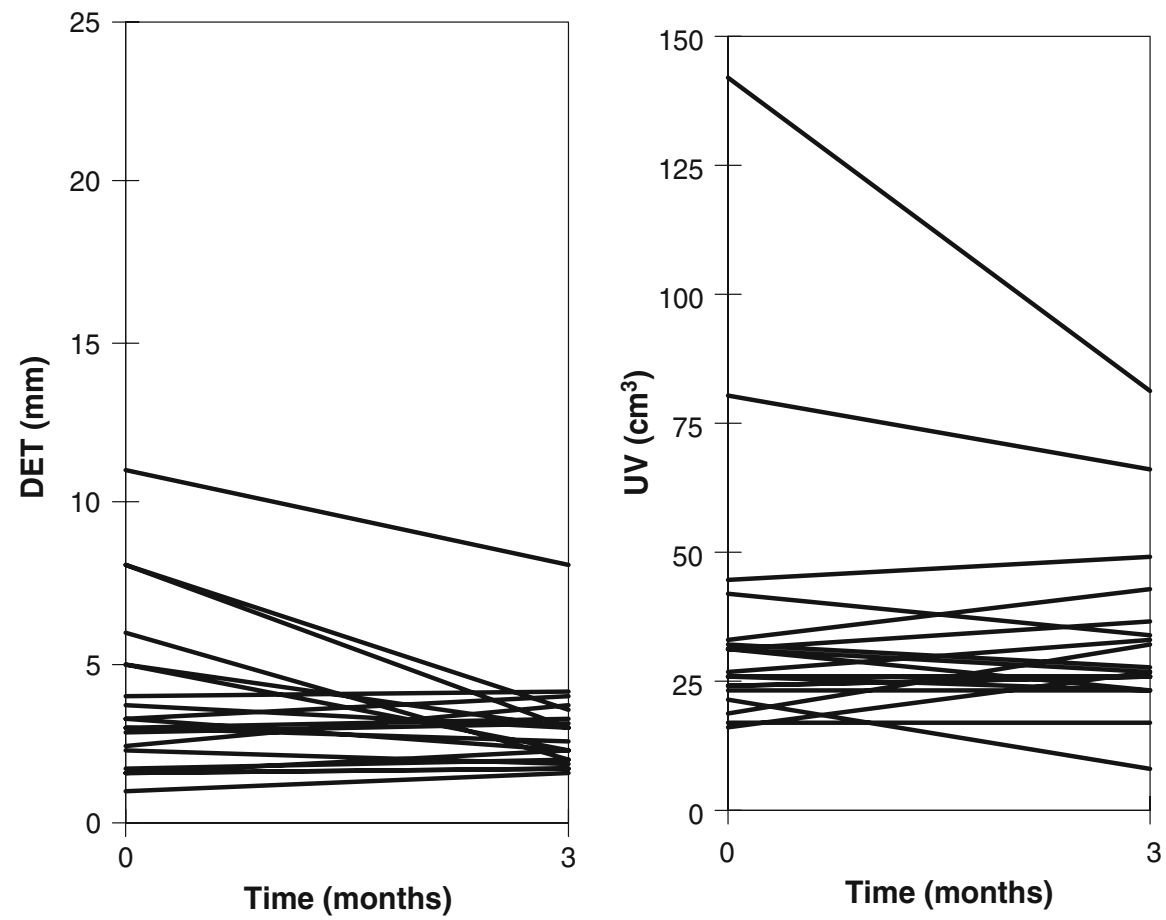
Table 2 Changes in pelvic abnormalities on TVUS after 3 months of therapy
Abbreviations: TVUS

transvaginal ultrasonography

\begin{tabular}{lllll}
\hline Type of abnormality & \multicolumn{3}{l}{ No. of patients with abnormality } & \\
\cline { 2 - 5 } & Baseline & 3 Months & \\
\cline { 2 - 5 } & & Increased/new & No change & Decreased/disappeared \\
\hline Endometrial cysts & 5 & $0 / 0$ & 5 & 0 \\
Polyps & 2 & $1 / 0$ & 0 & $1 / 0$ \\
Fibroids & 4 & $1 / 0$ & 2 & $1 / 0$ \\
Hydrosalpinx & 1 & $1 / 0$ & 0 & $0 / 0$ \\
Ovarian metastasis & 1 & $0 / 0$ & 0 & $1 / 0$ \\
\hline
\end{tabular}

not change in size or even decreased after 3 months of fulvestrant therapy. This is in accordance to what has been reported in case reports on the efficacy of fulvestrant in hormone receptor-positive uterine cancer [26, 27]. Furthermore, it is noteworthy that there were no episodes of vaginal bleeding reported in our series. The absence of abnormal vaginal bleeding is of clinical significance since most interventions such as hysteroscopy and endometrial biopsy are performed when patients report vaginal bleeding under anti-hormonal therapy [29-31]. The absence of vaginal bleeding with an estrogen antagonist may then translate into fewer gynecological interventions in breast cancer patients.

It is ideal that improvements in disease outcome with endocrine therapy for breast cancer patients would be associated with lesser side effects. The decrease in DET and UV seen in our study is consistent with the pharmacologic property of fulvestrant as an estrogen-receptor antagonist that is devoid of agonist activity on the uterus of postmenopausal breast cancer patients. The absence of a stimulatory effect on the endometrium can be considered as one of the benefits gained with fulvestrant therapy.

Acknowledgments We thank all the physicians, trial nurses, pharmacists and secretaries who assisted us and most especially the patients who kindly agreed to participate in this study. This research was conducted with support from the Investigator-Sponsored Study Program of AstraZeneca. AstraZeneca kindly provided fulvestrant (Faslodex) for the study patients, but was not involved in the study design, in the collection, analysis, and interpretation of data, in the writing of the manuscript nor in the decision to submit the manuscript for publication. AstraZeneca provided an unrestricted grant which was used for a Ph.D. stipend (LM).

\section{References}

1. Neven P, De Muylder X, Van Belle Y et al (1989) Tamoxifen and the uterus and endometrium. Lancet 1:375. doi:10.1016/S01406736(89)91741-8

2. Fornander T, Rutquivist LE, Wilking N (1991) Effects of tamoxifen on the female genital tract. Ann N Y Acad Sci 622:469-476. doi:10.1111/j.1749-6632.1991.tb37890.x

3. Kedar RP, Bourne TH, Powles TJ et al (1994) Effects of tamoxifen on the uterus and ovaries of postmenopausal women in a randomised breast cancer prevention trial. Lancet 343:13181321. doi:10.1016/S0140-6736(94)92466-X
4. Fisher B, Constantino JP, Wickerham DL et al (1998) Tamoxifen for prevention of breast cancer. Report of the national surgical adjuvant breast and bowel project P-1 study. J Natl Cancer Inst 90:1371-1388. doi:10.1093/jnci/90.18.1371

5. Morales L, Neven P, Paridaens R (2005) Choosing between an aromatase inhibitor and tamoxifen in the adjuvant setting. Curr Opin Oncol 17:559-565. doi:10.1097/01.cco.0000180434. 31991.bf

6. Poole R, Paridaens R (2007) The use of third-generation aromatase inhibitors and tamoxifen in the adjuvant treatment of postmenopausal patients with hormone-dependent breast cancer: evidence based review. Curr Opin Oncol 19:564-572

7. Thuerlimann B, Koeberle D, Senn HJ (2007) Guidelines for the adjuvant treatment of postmenopausal women with endocrineresponsive breast cancer: past, present and future recommendations. Eur J Cancer 43:46-52. doi:10.1016/j.ejca.2006.09.003

8. Morales L, Timmerman D, Neven P et al (2005) Third generation aromatase inhibitors may prevent endometrial growth and reverse tamoxifen-induced uterine changes in postmenopausal breast cancer patients. Ann Oncol 16:70-74. doi:10.1093/annonc/mdi021

9. Garrone O, Mezi S, Occelli M (2003) Reversal of tamoxifen induced endometrial modifications by switching to letrozole in early breast cancer patients: a prospective TVUS study. Ann Oncol 14:s22

10. Duffy S, Jackson TL, Lansdown M et al (2006) The ATAC ('Arimidex', Tamoxifen, Alone or in Combination) adjuvant breast cancer trial: first results of the endometrial sub-protocol following 2 years of treatment. Hum Reprod 21:545-553. doi: 10.1093/humrep/dei322

11. Gerber B, Krause A, Reimer T et al (2006) Anastrozole versus tamoxifen treatment in postmenopausal women with endocrineresponsive breast cancer and tamoxifen-induced endometrial pathology. Clin Cancer Res 12:1245-1250. doi:10.1158/10780432.CCR-05-0225

12. Garuti G, Cellani F, Centinaio G et al (2006) Prospective endometrial assessment of breast cancer patients treated with third generation aromatase inhibitors. Gynecol Oncol 103:599-603. doi:10.1016/j.ygyno.2006.04.004

13. Markovitch O, Tepper R, Fishman A et al (2007) Aromatase inhibitors reverse tamoxifen induced endometrial ultrasonographic changes in postmenopausal breast cancer patients. Breast Cancer Res Treat 101:185-190. doi:10.1007/s10549-006-9285-x

14. Bertelli G, Hall E, Ireland E et al (2007) Endometrial status in the Intergroup Exemestane Study (IES) up to 2 years post-treatment. Breast Cancer Res Treat 106:s111

15. Kieback DG, Harbeck N, Bauer W et al (2008) Endometrial safety of cross-over treatment with tamoxifen followed by exemestane. Eur J Cancer 6:s198

16. Boruban MC, Altundag K, Kilic GS, Blankstein J (2008) From endometrial hyperplasia to endometrial cancer: insight into the biology and possible medical preventive measures. Eur J Cancer Prev 17:133-138 
17. Robertson JFR (2001) Faslodex (ICI 182, 780), a novel estrogen receptor downregulator-future possibilities in breast cancer. J Steroid Biochem Mol Biol 79:209-212. doi:10.1016/S09600760(01)00138-8

18. Howell A, Pippen J, Elledge RM et al (2005) Fulvestrant versus anastrozole for the treatment of advanced breast carcinoma. Cancer 104:236-239. doi:10.1002/cncr.21163

19. Perey L, Paridaens R, Hawle H et al (2007) Clinical benefit of fulvestrant in postmenopausal women with advanced breast cancer and primary or acquired resistance to aromatase inhibitors: final results of phase II Swiss Group for Clinical Cancer Research Trial (SAKK 21/00). Ann Oncol 18:64-69. doi:10.1093/annonc/mdl341

20. Neven P, Paridaens R, Pelgrims G et al (2008) Fulvestrant (Faslodex) in advanced breast cancer: clinical experience from a Belgian cooperative study. Breast Cancer Res Treat 109:59-65. doi:10.1007/s10549-007-9628-2

21. Robertson JF, Come SE, Jones SE et al (2005) Endocrine treatment options for advanced breast cancer-the role of fulvestrant. Eur J Cancer 41:346-356. doi:10.1016/j.ejca.2004.07.035

22. Chia S, Gradishar W, Mauriac L et al (2008) Double-blind, randomized placebo controlled trial of fulvestrant compared with exemestane after prior non-steroidal aromatase inhibitor therapy in postmenopausal women with hormone receptor-positive, advanced breast cancer: results from EFECT. J Clin Oncol 26:1664-1670. doi:10.1200/JCO.2007.13.5822

23. Gaducci A, Cosio S, Genazzani AR (2004) Use of estrogen antagonists and aromatase inhibitors in breast cancer and hormonally sensitive tumors of the uterine body. Curr Opin Investig Drugs 5:1031-1044
24. Addo S, Yates RA, Laight A (2002) A phase I trial to assess the pharmacology of the new oestrogen receptor antagonist fulvestrant on the endometrium in healthy postmenopausal volunteers. Br J Cancer 87:1354-1359. doi:10.1038/sj.bjc.6600644

25. Donnez J, Vivancos BH, Kudela M et al (2003) A randomized, placebo-controlled, dose-ranging trial comparing fulvestrant with goserelin in premenopausal patients with uterine fibroids awaiting hysterectomy. Fertil Steril 79:1380-1389. doi:10.1016/S00150282(03)00261-9

26. Lux M, Wenkel E, Beckmann K et al (2006) Fulvestrant: a further treatment option for patients with metastatic uterine cancer? Onkologie 29:577-580. doi:10.1159/000096541

27. Hoffman MA, Khan A (2006) Durable response of metastatic endometrial carcinoma to treatment with fulvestrant (Faslodex) after prior progestin and anastrozole therapy. Gynecol Oncol 100:439-441. doi:10.1016/j.ygyno.2005.09.011

28. World Medical Association (2008) Review of the declaration of Helsinki. http://www.wma.net

29. Neven P, Vergote I (1998) Should tamoxifen users be screened for endometrial lesions? Lancet 351:155-157. doi:10.1016/ S0140-6736(05)78216-7

30. Berlière M, Galant C, Charles A et al (2002) Endometrial evaluation is a very important tool in the management of breast cancer patients. Eur J Cancer 38(suppl. 6):67-68. doi:10.1016/ S0959-8049(02)00292-7

31. Morales L, Paridaens R, Timmerman D, Neven P (2006) Aromatase inhibitors and postmenopausal breast cancer patients with tamoxifen-induced endometrial pathology. Clin Cancer Res 12:5603. doi:10.1158/1078-0432.CCR-06-0897 